\title{
УДК $622.7+541.183+66-5$ \\ УСТАНОВЛЕНИЕ ТЕХНОЛОГИЧЕСКИХ ЗАВИСИМОСТЕЙ РАБОТЫ КОНЦЕНТРАТОРА KC-CVD6 С ПОМОЩЬЮ МЕТОДА ГРУППОВОГО УЧЕТА АРГУМЕНТОВ
}

\author{
Пелих Владислав Вадимович1, \\ salov@istu.edu
}

\author{
Салов Валерий Михайлович2, \\ salov@istu.edu
}

Бурдонов Александр Евгеньевич²,
slimbul@inbox.ru

Лукьянов Никита Дмитриевич2, Lukyanov.n@gmail.com

1000 «ФЛСмидт Рус» в г. Иркутске,

Россия, 664007, г. Иркутск, ул. Октябрьской Революции, $1 / 4$.
2 Иркутский национальный исследовательский технический университет, Россия, 664074, г. Иркутск, ул. Лермонтова, 83.

\begin{abstract}
Актуальность исследования обусловлена получением новых знаний об оптимизации технологических параметров работы центробежных концентраторов, эксплуатируемых по технологии Knelson CVD, а именно: настройка частоты открытия клапанов и время, в течение которого клапаны остаются открытыми.

Цель: статистический анализ результатов полупромышленных исследований применимости технологии Knelson CVD на различных продуктах фабрики с помощью метода группового учета аргументов для получения зависимостей между выходом концентра и настраиваемыми параметрами, позволяющими проводить предварительные расчеты по эфрфективности внедрения данной технологии на обогатительных предпиятиях.

объекты: продукты, полученные в результате работы гидроциклонов, а также технологические параметры работы центробежных концентраторов.

Методы: общие методы математической статистики, в частности методы регрессионного анализа, направленные на построение статистически значимых моделей, описывающих зависимость какой-либо переменной от множества регрессоров. Также, наряду с классическим аппаратом регрессионного анализа, применяется метод группового учета аргументов, основная идея которого заключается в построении множества моделей заданного класса и выборе среди них оптимальной. Результаты. Разработана эфффективная методика обработки результатов тестов, проведенных на обогатительном оборудовании, базирующаяся на методе группового учета аргументов. На основании полученных данных построены статистически значимые модели, описывающие зависимость содержания в хвостах и концентратах по ценным компонентам от различных настраиваемых параметров оборудования, обоснована их эффрективность, позволяющая применять данные модели в дальнейших исследованиях по применению CVD технологии. Продемонстрировано применение метода группового учета аргументов на примере построения полиномиальных регрессионных моделей, содержащих нелинейные комбинации регрессоров.
\end{abstract}

\section{Ключевые слова:}

Knelson CVD, гравитационное обогащение, золото, свинец, извлечение, хвосты, технология.

\section{Введение}

Как уже отмечалось во многих публикациях [1-5], связанных с горно-обогатительной отраслью, минерально-сырьевая база в нашей стране и в мире постоянно меняется в связи с развитием научно-технического прогресса. В связи с чем переработка полезных ископаемых, в том числе полиметаллических руд, с применением устаревшего оборудования, используемого на многих обогатительных предприятиях, становиться нерентабельной.

Обеднение и снижение качества руд стимулирует применение все более сложных техникотехнологических процессов, видов оборудования, реагентов, в результате чего увеличивается себестоимость перерабатываемой продукции, опережая рыночные цены на конечные изделия, снижая конкурентоспособность и инвестиционную привлекательность предприятия. Оптимальным решением возникшей проблемы является изменение схем переработки руд и включение в них современного, высокоэффективного оборудования [6-8].

Одним из предприятий, требующих нового технологического подхода, является Ново-Широкинский комбинат, работающий на золотополиметаллическом месторождении в Восточном Забайкалье.

Основными производственными продуктами организации являются цинковый и свинцовый концентраты с годовым объемом выпуска в 9 и 25 т соответственно. Кроме того, «Новоширокинский рудник» по итогам января-июня 2018 г. произвело 61,027 тыс. унций золотого эквивалента. В первом полугодии 2018 г. коэффициент извлечения металла из руды был на уровне $80,3 \%$. В тот же период прошлого года коэффициент составлял 85,2\%.

Тем не менее существующа схема гравитационного обогащения на отсадочных машинах показывает низкую эффективность, по имеющимся данным: 
- первая стадия отсадки (цикл измельчения в мельнице мокрого полусамоизмельчения - МПСИ) при выходе 5-7 \% дает среднее извлечение золота $8 \%$, содержание 1,5-2 г/т, извлечение свинца 5-6\%, содержание свинца 2,5\%.

- вторая стадия МОТ (цикл измельчения в шаровой мельнице с центральной разгрузкой - МЩЦ) при выходе $30 \%$ дает среднее извлечение золота $39 \%$, содержание 6-10 г/т, извлечение свинца 28-30\%, содержание свинца 1,3-3\%.

Представленные результаты схемы гравитационного обогащения на отсадочных машинах показывают высокий выход концентрата и низкую концентрацию целевых компонентов. Анализ мирового опыта переработки руд показывает, что использование современных технологий, в частности технологии CVD, может повысить технологические показатели и увеличить эффективность работы фабрики. Комплекс проведённых лабораторных исследований в НИиПИ TOMС (Институт) показал хорошие перспективы для внедрения данной технологии [9].

Необходимо отметить, что постоянные работы по настройке и оптимизации работы горнообогатительного оборудования являются дорогостоящими [10-12]. Тем самым, получение значений и статистических зависимостей позволяет проводить предварительные расчеты по эффективности внедрения новых технологий на таких предприятиях.

Целью данной статьи является статистический анализ результатов полупромышленных исследований применимости технологии Knelson CVD на различных продуктах фабрики с помощью метода группового учета аргументов.

\section{Методика и результаты проведения} полупромышленных испытаний

Полупромышленные исследования проводились на нескольких продуктах фабрики: вторичный цикл измельчения на сливе МШЦ, слив гидроциклона второй стадии измельчения перед флотацией и на хвостах цинковой флотации. Данное решение принято с целью получения наиболее полной и объективной информации касательно эффективности использования данного типа оборудования.

В работе рассматривались результаты, полученные только на сливе гидроциклона.

Методика проведения испытаний строилась на нескольких этапах: один этап общий подготовительный и три этапа для каждого.

Методика проведения полупромышленных испытаний при подработке каждого технологического потока строилась на трёх этапах:

1. Определение оптимального расхода флюидизационной воды путём отбора проб на каждом режиме подачи флюидизационной воды.

2. Определение извлечения и степени концентрации золота и свинца при различных режимах выхода концентрата, значениях времени закрытия пережимных клапанов, при отборе проб технологических потоков, с определением фактического массового выхода концентрата.

3. Проведение серии испытаний на оптимальных настройках концентратора, определённых в ходе двух предыдущих этапов. Отбор всего объёма концентрата, отбор проб для подтверждения результатов предыдущих испытаний.

Подробное описание методики проведения испытаний представлено в работе [13].

При проведении испытаний на сливе гидроциклона подготовка питания осуществлялась по схеме, изображённой на рис. 1. Из ёмкости слива гидроциклона питание насосом перекачивалось по трубопроводу на тройник, один поток которого направлялся в схему фабрики и регулировался задвижкой, второй поток уходил в схему питания установки, тем самым обеспечивалась приемлемая стабильность питания.

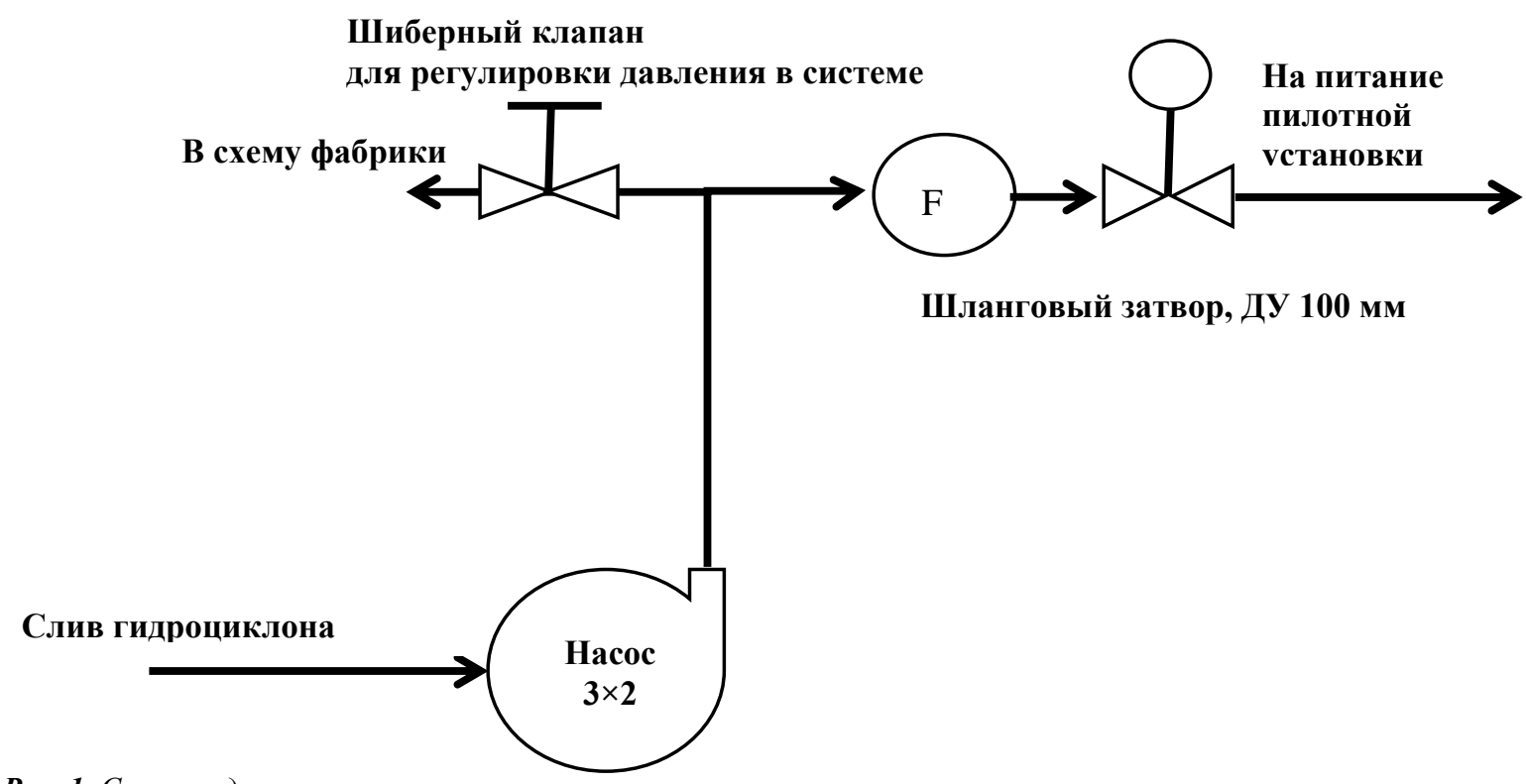

Pис. 1. Схема подготовки питания

Fig. 1. Nutrition training scheme 


\section{Первый этап проведения испытаний}

Целью первого этапа испытаний был подбор параметров расхода флюидизационной воды на основе оценки содержания ценного компонента в продуктах концентратора. Эксперимент (Этап 1) проходил при следующих условиях: расход флюидизационной воды варьировался от 25 до 40 л/м (интервал 5 л/м), скорость вращения ротора 920 об/мин/75G, время «открытия клапана» 0,325 с, время «закрытия клапана» 6 с, Давление воздуха в пережимных клапанах 55 PSI, Длительность эксперимента 1 час. Результаты испытаний представлены в табл. 1.

Таблица 1. Результаты определения оптимального расхода флюидизачионной воды на сливе гидроциклона

Table 1. Results of determining the optimal flow rate of fluidized water at the sink of a hydrocyclone

\begin{tabular}{|c|c|c|c|c|c|}
\hline \multicolumn{6}{|c|}{ По золоту/By gold } \\
\hline \multirow{2}{*}{ 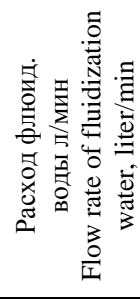 } & \multirow{2}{*}{ 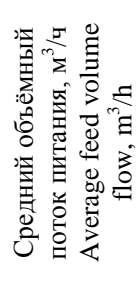 } & \multirow{2}{*}{ 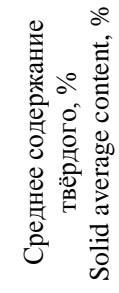 } & \multicolumn{3}{|c|}{$\begin{array}{l}\text { Содержание золота, г/ } \\
\text { Gold content, g/t }\end{array}$} \\
\hline & & & 惫总 & 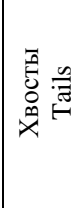 & 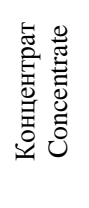 \\
\hline 25 & 4 & 30 & 1,6 & 1,2 & 2,4 \\
\hline 30 & 4 & 30 & 2,4 & 1,8 & 2,8 \\
\hline 35 & 3,5 & 31 & 1,6 & 1,2 & 2,4 \\
\hline 40 & 5 & 32 & 1,8 & 1,4 & 3,2 \\
\hline \multicolumn{6}{|c|}{ По свинцу/By lead } \\
\hline \multirow{2}{*}{ 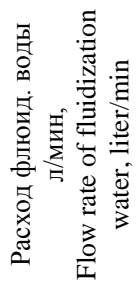 } & \multirow{2}{*}{ 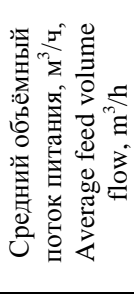 } & \multirow{2}{*}{ 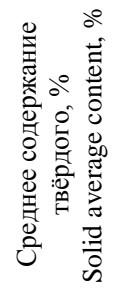 } & \multicolumn{3}{|c|}{$\begin{array}{c}\text { Массовая доля } \\
\text { свинца, \% } \\
\text { Lead mass fraction, \% }\end{array}$} \\
\hline & & & $\begin{array}{l}\text { 莹 } \\
\text { 总 } \\
\text { 㤩 }\end{array}$ & 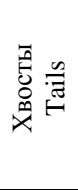 & 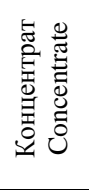 \\
\hline 25 & 4 & 30 & 0,79 & 0,77 & 2,16 \\
\hline 30 & 4 & 30 & 0,57 & 0,49 & 1,83 \\
\hline 35 & 3,5 & 31 & 0,58 & 0,5 & 1,77 \\
\hline 40 & 5 & 32 & 0,61 & 0,55 & 1,95 \\
\hline
\end{tabular}

\section{Второй этап проведения испытаний}

Целью второго этапа являлось установление зависимостей выход-концентрат и выход-извлечение, на основании данных, полученных в результате эксперимента с различным выходом концентрата, а также выбор наиболее эффективных режимов для длительных испытаний. Длительность каждого эксперимента составляла 1 час. Варьировалось значение времени закрытия пережимных клапанов. Эксперимент проходил при тех же условиях.

На рис. 2, 3 представлены графики, иллюстрирующие зависимости, полученные в результате испытаний (табл. 3).
Таблица 2. Определение выхода концентрата на сливе гидрочиклона в зависимости от настроек пережимных клапанов

Table 2. Determination of concentrate output at hydrocyclone discharge depending on the pinch valve settings

\begin{tabular}{|c|c|c|c|c|}
\hline \multicolumn{5}{|c|}{ По золоту/By gold } \\
\hline \multirow{2}{*}{$\begin{array}{c}\text { Время } \\
\text { «открытия } \\
\text { клапана», с } \\
\text { PV open } \\
\text { time, s }\end{array}$} & \multirow{2}{*}{$\begin{array}{c}\text { Время } \\
\text { «закрытия } \\
\text { клапана», с } \\
\text { PV closing } \\
\text { time, } \mathrm{s}\end{array}$} & \multicolumn{3}{|c|}{$\begin{array}{c}\text { Содержание золота, г/т } \\
\text { Gold content, g/t }\end{array}$} \\
\hline & & $\begin{array}{l}\text { Питание } \\
\text { Nutrition }\end{array}$ & $\begin{array}{l}\text { Хвосты } \\
\text { Tails }\end{array}$ & $\begin{array}{l}\text { Концентрат } \\
\text { Concentrate }\end{array}$ \\
\hline 0,325 & 4 & 2 & 1,6 & 3,4 \\
\hline 0,325 & 8 & 1,8 & 1,4 & 4,6 \\
\hline 0,325 & 12 & 1,8 & 1,6 & 6 \\
\hline 0,325 & 16 & 2,2 & 1,8 & 5,8 \\
\hline 0,325 & 24 & 2 & 1,6 & 5,4 \\
\hline 0,325 & 32 & 1,4 & 1,3 & 4,3 \\
\hline \multicolumn{5}{|c|}{ По свинцу/By lead } \\
\hline \multirow{2}{*}{$\begin{array}{c}\text { Время } \\
\text { «открытия } \\
\text { клапана», с } \\
\text { PV open } \\
\text { time, s }\end{array}$} & \multirow{2}{*}{$\begin{array}{c}\text { Время } \\
\text { «закрытия } \\
\text { клапана», с } \\
\text { PV closing } \\
\text { time, s }\end{array}$} & \multicolumn{3}{|c|}{$\begin{array}{c}\text { Массовая доля свинца, \% } \\
\text { Lead mass fraction, } \%\end{array}$} \\
\hline & & $\begin{array}{l}\text { Питание } \\
\text { Nutrition }\end{array}$ & $\begin{array}{l}\text { Хвосты } \\
\text { Tails }\end{array}$ & $\begin{array}{l}\text { Концентрат } \\
\text { Concentrate }\end{array}$ \\
\hline 0,325 & 4 & 0,63 & 0,34 & 1,4 \\
\hline 0,325 & 8 & 0,65 & 0,4 & 2,11 \\
\hline 0,325 & 12 & 1,3 & 0,94 & 4,75 \\
\hline 0,325 & 16 & 0,41 & 0,25 & 2,45 \\
\hline 0,325 & 24 & 0,35 & 0,28 & 2,15 \\
\hline 0,325 & 32 & 0,35 & 0,27 & 2,6 \\
\hline
\end{tabular}

Таблица 3. Определение выхода и извлечения $A u$ и $\mathrm{Pb}$ кониентратов на сливе гидроциклона в зависимости от настроек пережимных клапанов

Table 3. Determination of the yield and extraction of Au and $\mathrm{Pb}$ concentrates at hydrocyclone discharge depending on the settings of the pinch valves

\begin{tabular}{|c|c|c|c|c|c|c|}
\hline \multirow{2}{*}{ 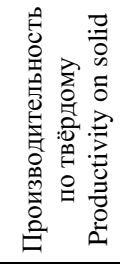 } & \multirow{2}{*}{ 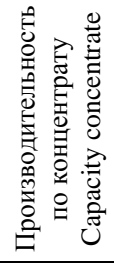 } & \multirow{3}{*}{ 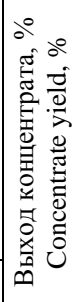 } & \multicolumn{2}{|c|}{$\begin{array}{c}\text { Извлечение в } \\
\text { концентрат, \%, } \\
\text { Extraction in } \\
\text { concentrate, \% }\end{array}$} & \multicolumn{2}{|c|}{$\begin{array}{c}\text { Коэффициент } \\
\text { концентрации } \\
\text { Concentration } \\
\text { ratio }\end{array}$} \\
\hline & & & $\mathrm{Au}$ & $\mathrm{Pb}$ & $\mathrm{Au}$ & $\mathrm{Pb}$ \\
\hline \multicolumn{2}{|c|}{$\mathrm{T} / \mathrm{u} / \mathrm{t} / \mathrm{h}$} & & & & & \\
\hline 1981,72 & 541,0 & 27,3 & 46,4 & 60,61 & 1,7 & 2,2 \\
\hline 1928,16 & 281,1 & 14,6 & 37,2 & 47,31 & 2,51 & 3,2 \\
\hline 2035,28 & 189,8 & 9,34 & 31,1 & 34,1 & 3,31 & 3,6 \\
\hline 2035,28 & 143,3 & 7,1 & 18,6 & 42,1 & 2,61 & 5,9 \\
\hline 1928,16 & 96,2 & 4,9 & 13,5 & 30,6 & 2,7 & 6,1 \\
\hline 2229,435 & 72,4 & 3,2 & 9,9 & 24,1 & 3,1 & 7,4 \\
\hline
\end{tabular}

\section{Третий этап проведения испытаний}

Целью третьего этапа являлось подтверждение полученных в результате предыдущих испытаний значений, а также накопление концентрата для проведения последующих испытаний по доводке (табл. 4, 5).

Длительность эксперимента 3 часа. Средний объёмный поток питания 5 м³/ч. 


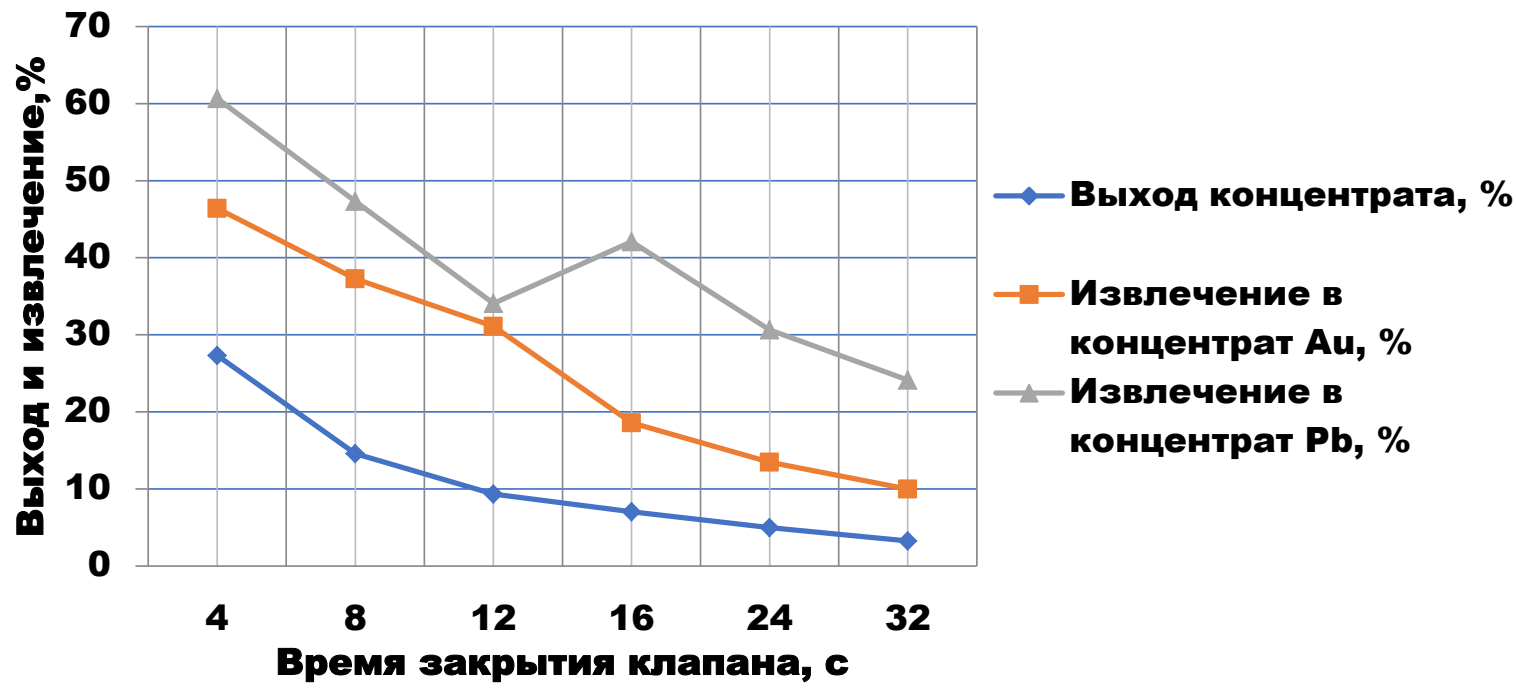

Рис. 2. Кривая выхода концентрата/извлечения

Fig 2. Concentrate yield/extraction curve

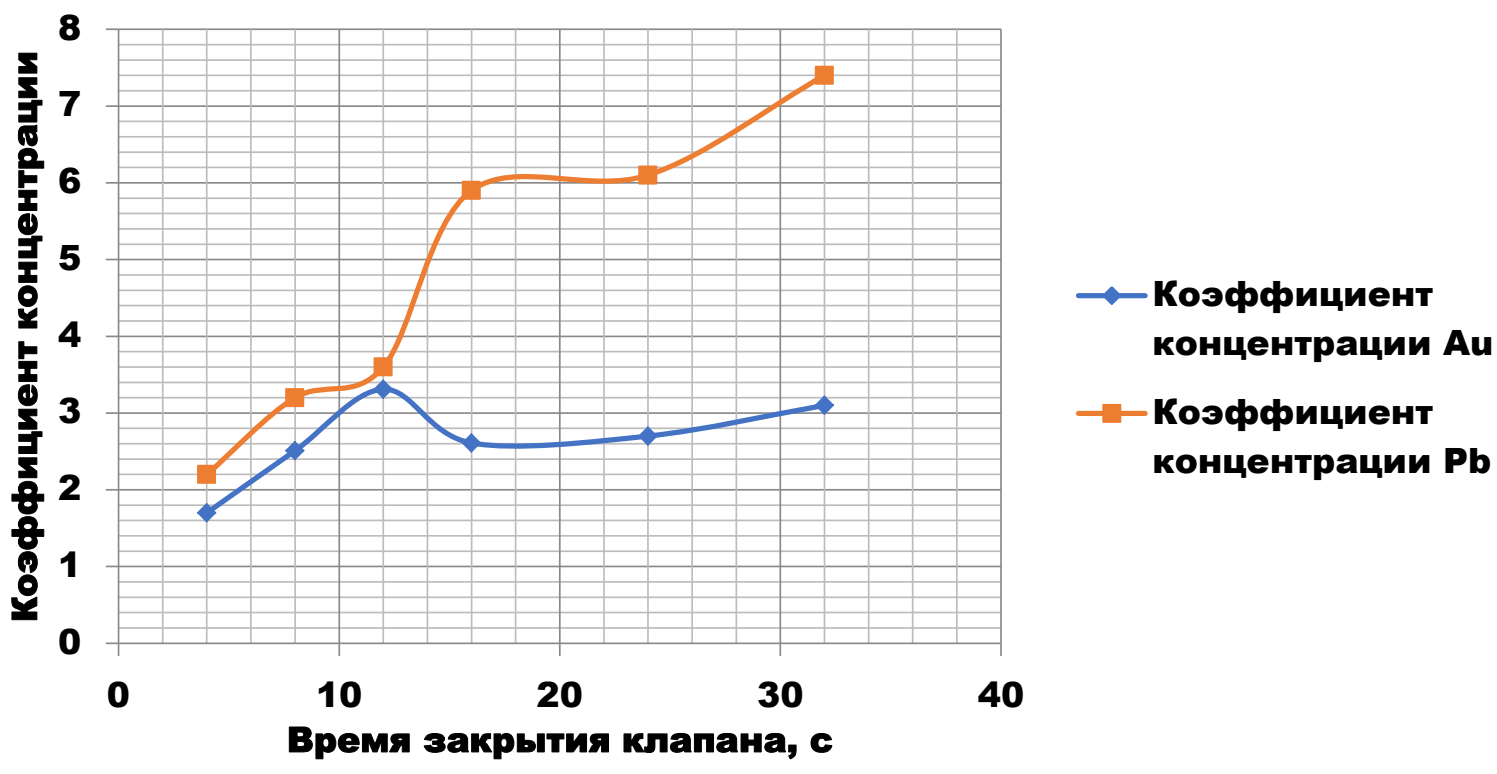

Pис. 3. Кривые коэффициентов концентрации

Fig. 3. Concentration curves

Таблица 4. Результаты испытаний по накоплению концентрата на выбранных режсимах Table 4. Test results on concentrate accumulation on the selected modes

\begin{tabular}{|c|c|c|c|c|c|}
\hline \multicolumn{6}{|c|}{ По золоту/By gold } \\
\hline \multirow{2}{*}{$\begin{array}{c}\text { Время «открытия клапана», с } \\
\text { PV open time, s }\end{array}$} & \multirow{2}{*}{$\begin{array}{c}\text { Время «закрытия клапана», с } \\
\text { PV closing time, s }\end{array}$} & \multicolumn{3}{|c|}{$\begin{array}{c}\text { Содержание золота, г/т } \\
\text { Gold content, g/t }\end{array}$} & \multirow{2}{*}{$\begin{array}{c}\text { Масса накопленного концентрата, } \\
\text { кг } \\
\text { Mass of accumulated concentrate, } \mathrm{kg}\end{array}$} \\
\hline & & $\begin{array}{l}\text { Питание } \\
\text { Nutrition }\end{array}$ & $\begin{array}{c}\text { Хвосты } \\
\text { Tails }\end{array}$ & $\begin{array}{l}\text { Концентрат } \\
\text { Concentrate }\end{array}$ & \\
\hline 0,325 & 4 & 2,1 & 1,1 & 5,6 & 477 \\
\hline 0,325 & 12 & 2,2 & 1,3 & 8,98 & 387 \\
\hline 0,325 & 24 & 2,2 & 1,8 & 9,6 & 180 \\
\hline \multicolumn{6}{|c|}{ По свинцу/By lead } \\
\hline \multirow{2}{*}{$\begin{array}{c}\text { Время «открытия клапана», с } \\
\text { PV open time, s }\end{array}$} & \multirow{2}{*}{$\begin{array}{c}\text { Время «закрытия клапана», с } \\
\text { PV closing time, s }\end{array}$} & \multicolumn{3}{|c|}{$\begin{array}{c}\text { Массовая доля свинца, \% } \\
\text { Lead mass fraction, \% }\end{array}$} & \multirow{2}{*}{$\begin{array}{c}\text { Масса накопленного концентрата, } \\
\text { кг } \\
\text { Mass of accumulated concentrate, } \mathrm{kg}\end{array}$} \\
\hline & & \begin{tabular}{l|} 
Питание \\
Nutrition \\
\end{tabular} & $\begin{array}{c}\text { Хвосты } \\
\text { Tails } \\
\end{array}$ & $\begin{array}{l}\text { Концентрат } \\
\text { Concentrate }\end{array}$ & \\
\hline 0,325 & 4 & 0,41 & 0,23 & 1,73 & 477 \\
\hline 0,325 & 12 & 0,19 & 0,23 & 1,94 & 387 \\
\hline 0,325 & 24 & 0,26 & 0,17 & 1,66 & 180 \\
\hline
\end{tabular}


Таблица 5. Результаты испьтаний по извлечению концентрата

Table 5. Test results on concentrate extraction

\begin{tabular}{|c|c|c|c|c|c|c|c|c|}
\hline \multirow[t]{2}{*}{$\begin{array}{c}\text { Время «от- } \\
\text { крытия кла- } \\
\text { пана», c } \\
\text { PV open time, } \\
\text { s }\end{array}$} & \multirow[t]{2}{*}{$\begin{array}{c}\text { Время «за- } \\
\text { крытия кла- } \\
\text { пана», с } \\
\text { PV closing } \\
\text { time, s }\end{array}$} & \multirow[t]{2}{*}{$\begin{array}{c}\text { Производительность } \\
\text { по твёрдому, т/ч } \\
\text { Productivity on solid, } \\
\text { t/h }\end{array}$} & \multirow[t]{2}{*}{$\begin{array}{c}\text { Производительность по } \\
\text { концентрату, т/ч } \\
\text { Capacity concentrate, t/h }\end{array}$} & \multirow[t]{2}{*}{$\begin{array}{c}\text { Выход } \\
\text { концентрата, \% } \\
\text { Concentrate } \\
\text { yield, } \%\end{array}$} & \multicolumn{2}{|c|}{$\begin{array}{l}\text { Извлечение в } \\
\text { концентрат, \% } \\
\text { Extraction into } \\
\text { concentrate, } \%\end{array}$} & \multicolumn{2}{|c|}{$\begin{array}{c}\text { Коэффициент } \\
\text { концентрации, } \\
\text { Concentration } \\
\text { ratio }\end{array}$} \\
\hline & & & & & $\mathrm{Au}$ & $\mathrm{Pb}$ & $\mathrm{Au}$ & $\mathrm{Pb}$ \\
\hline 0,325 & 4 & 2148,12 & 477 & 22,2 & 59,21 & 93,69 & 2,66 & 4,21 \\
\hline 0,325 & 12 & 1652,48 & 193,5 & 11,7 & 47,79 & 119,5 & 4,08 & 10,2 \\
\hline 0,325 & 24 & 1755,76 & 90 & 5,1 & 22,36 & 32,72 & 4,36 & 6,38 \\
\hline
\end{tabular}

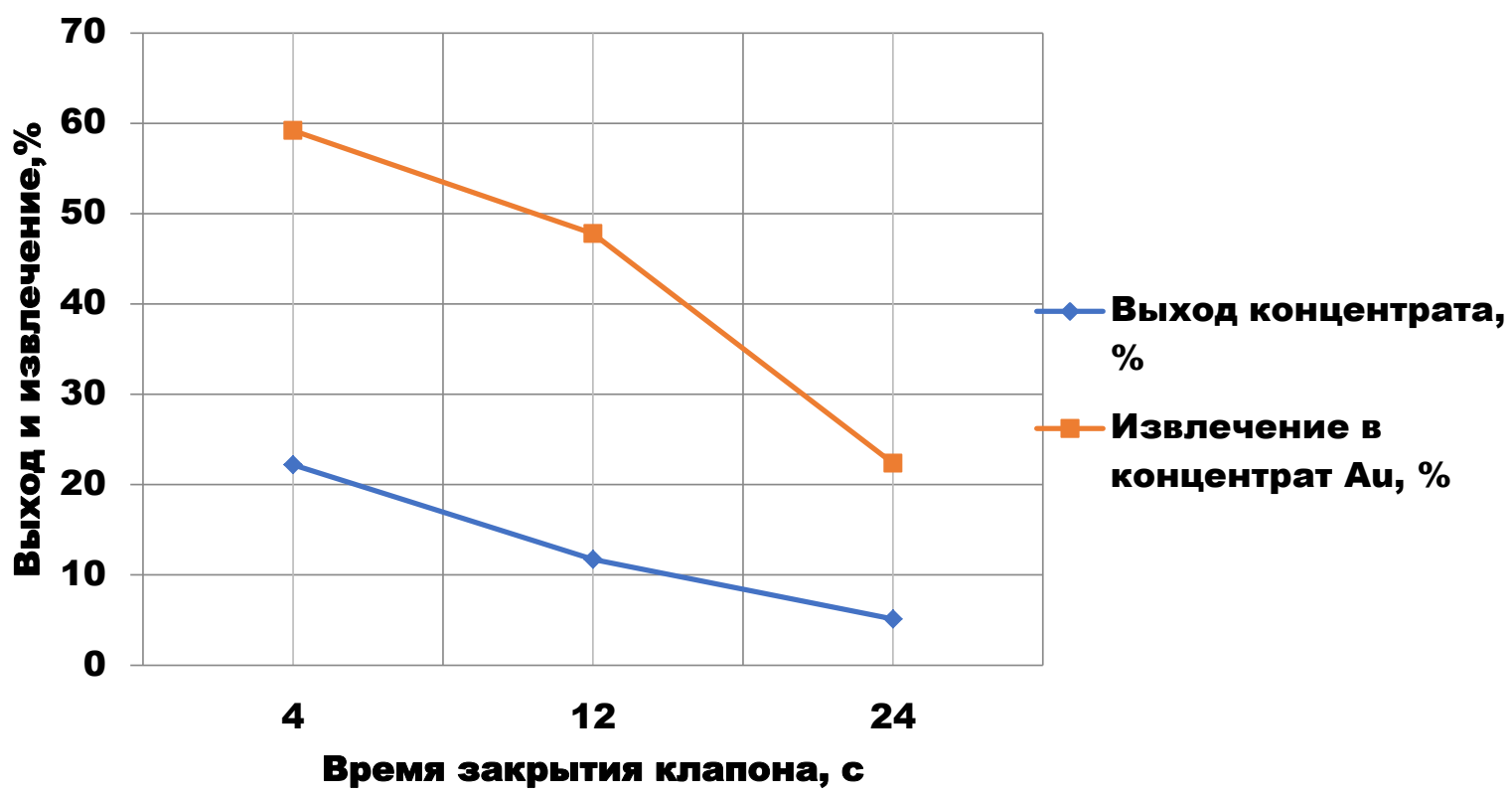

Рис. 4. Кривая выхода конщентрата/извлечения

Fig. 4. Concentrate yield/extraction curve

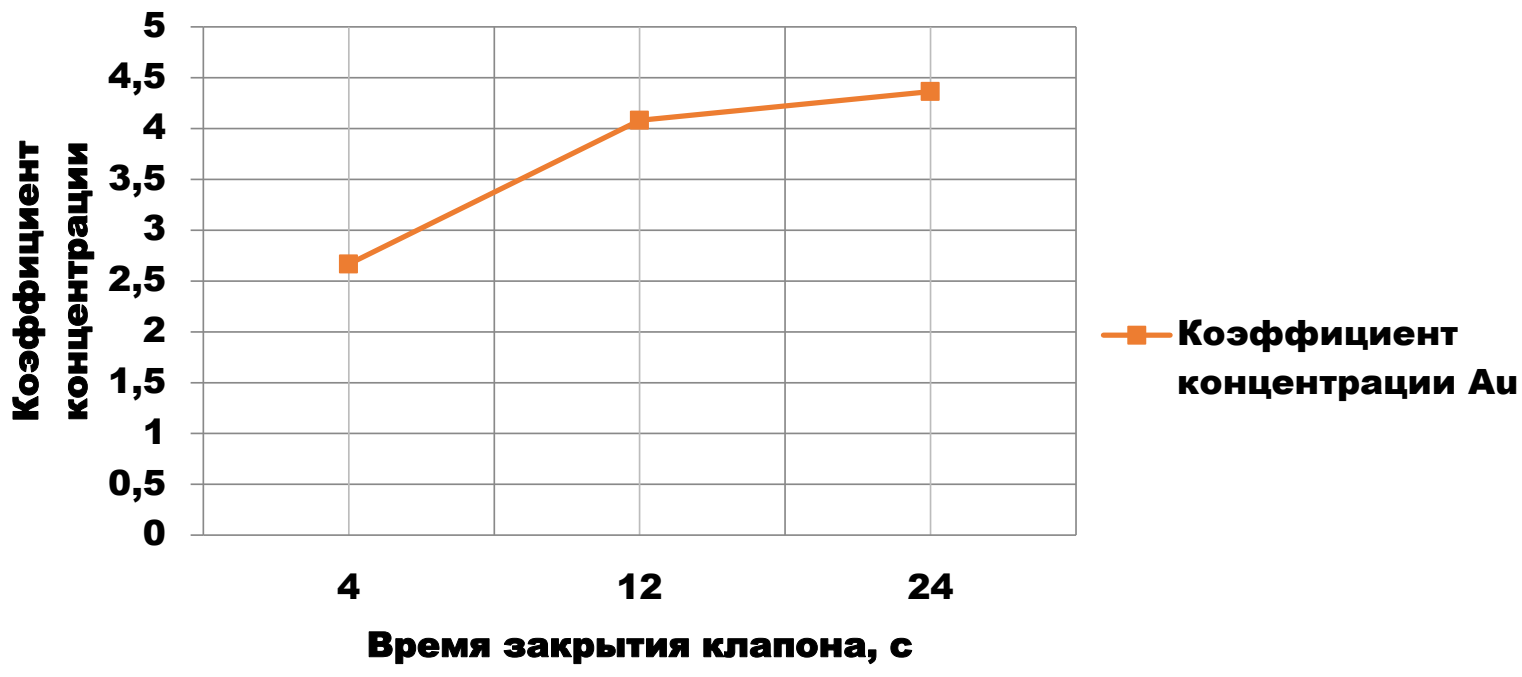

Pис. 5. Кривая коэффициента концентрации

Fig. 5. Concentration ratio curve

Во время проведения эксперимента все отсадочные машины фабрики были отключены. Плотность питания дополнительно не регулировалась, в ходе экспериментов производился отбор разгрузок концентратора с целью определения массового вы- хода концентрата. Отбор проб для определения содержания в технологических потоках пилотной установки осуществлялся через равные промежутки времени в течение всего теста, а также для каждого теста. 
Проведение тестов на сливе гидроциклона характеризовалось стабильными характеристиками плотности питания, что было обусловлено подачей питания под давлением. Поскольку вся гравитационная схема фабрики была отключена, плотность слива гидроциклона повысилась на 8 до 37 \%, тем не менее это значение осталось в пределах приемлемой плотности питания концентратора KC-CVD6. Для повышения точности получаемых данных замеры плотности происходили каждые 20 минут, после чего рассчитывался средний показатель. Учёт объёма производился по сумматору расходомера.

Установлено, что извлечение золота носит более предсказуемый характер по сравнению с тестами на сливе мельницы [14] и имеет практически прямую зависимость от выхода концентрата. В первую очередь это связано с более узким гранулометрическим составом и отсутствием циркуляционных нагрузок, а также с тем, что большая часть зёрен несущих целевых минералов уже вскрыта.

Укрупнённая оценка показала, что при выходе концентрата 10-20\% извлекается 30-50 \% золота. Коэффициент концентрации варьируется от 1,5 до 4. Результаты, полученные по свинцу в финальных (накопительных) тестах, нельзя принять как представительные, вероятно, на низких концентрациях процедура определения содержания по технологии лаборатории не даёт достаточной точности. Тем не менее, первичные часовые тесты выглядят представительными. Это связано с более высоким содержанием ценных компонентов в питании, что снижало погрешность. Установлено, что динамика извлечения по зависимости от выхода схожа с поведением золота, но по всем тестам в среднем выше на $15 \%$.

\section{Обработка результатов с помощью метода группового учета аргументов}

Проведем обработку полученных результатов, используя аппарат математической статистики. Для этого определим множество регрессоров (т. е. факторов, которыми можно управлять) и множество зависимых переменных. В нашем случае регрессорами являются: расход флюидизационной воды, обозначим как $x_{1}$, и время закрытия пережимного клапана, обозначим как $x_{2}$. В качестве зависимых переменных примем содержание концентрата по $\mathrm{Au}$, хвостов по $\mathrm{Au}$, содержание концентрата по $\mathrm{Pb}$ и хвостов по $\mathrm{Pb}$, обозначим данные величины как $y_{1}, y_{2}, y_{3}$ и $y_{4}$ соответственно.

Представим результаты проведенных тестов слива гидроциклона (табл. 6).

Таблица составлена из всех промежуточных таблиц и отсортирована по переменной $x_{2}$. Для повторяющихся значений регрессоров значения зависимых переменных взяты как среднее арифметическое.

Составим линейную регрессионную модель, описывающую зависимость какой-либо зависимой переменной от регрессоров по методике [14].

Будем искать зависимость параметра $y_{1}$ в виде линейной функции от настраиваемых параметров $x_{1}$ и $x_{2}$ :

$$
y_{1}=b_{0}+\sum_{i=1}^{2} b_{i} x_{i}
$$

Таблица 6. Сводная таблица результатов теста слива гидрочиклона

Table 6. Summary of the results of the hydrocyclone discharge test

\begin{tabular}{|c|c|c|c|c|c|}
\hline$x_{1}$ & $x_{2}$ & $y_{1}$ & $y_{2}$ & $y_{3}$ & $y_{4}$ \\
\hline 35 & 4 & 4,5 & 1,35 & 1,565 & 0,285 \\
\hline 25 & 6 & 2,4 & 1,2 & 2,16 & 0,77 \\
\hline 30 & 6 & 2,8 & 1,8 & 1,83 & 0,49 \\
\hline 35 & 6 & 2,4 & 1,2 & 1,77 & 0,5 \\
\hline 40 & 6 & 3,2 & 1,4 & 1,95 & 0,55 \\
\hline 35 & 8 & 4,6 & 1,4 & 2,11 & 0,4 \\
\hline 35 & 12 & 7,49 & 1,45 & 3,345 & 0,585 \\
\hline 35 & 16 & 5,8 & 1,8 & 2,45 & 0,25 \\
\hline 35 & 24 & 7,5 & 1,7 & 1,905 & 0,225 \\
\hline 35 & 32 & 4,3 & 1,3 & 2,6 & 0,27 \\
\hline
\end{tabular}

Найдем с помощью метода наименьших квадратов, что такая зависимость описывается следующей формулой:

$$
y_{1}=-0,846+0,124 x_{1}+0,092 x_{2} .
$$

При этом коэффициент детерминации $R^{2}$ для данной модели равен 0,31 , что не позволяет её использовать даже для проведения грубых, предварительных расчётов. Аналогичные исследования, проведенные для переменных $y_{2}, y_{3}$ и $y_{4}$, дают $R^{2}$, равный 0,037 , 0,48 и 0,162 , соответственно. Полученные результаты позволяют выдвинуть две конкурирующих статистических гипотезы:

1. Указанные переменные не состоят в статистически значимой зависимости от регрессоров.

2. Статистически значимая зависимость присутствует, но носит нелинейный характер.

Попытаемся подтвердить вторую гипотезу, используя метод группового учета аргументов (МГУА) [15], применение которого в данной работе обусловлено следующими причинами:

1. Ограниченное количество наблюдений $(N=10)$;

2. Доказательство отсутствия линейной зависимости между регрессорами и зависимыми переменными;

3. Отсутствие априорной информация о структуре искомой зависимости [16-18];

4. Простая реализация данного метода.

Опишем алгоритм применения МГУА для данной задачи. Основная идея данного метода заключается в построении множества моделей заданного класса, после чего происходит выбор наилучшей из них по определенному критерию. В данном случае будем рассматривать полиномиальные модели, общая формула которых записывается в следующем виде:

$$
y=b_{0}+\sum_{i=1}^{m} b_{i} x_{i}+\sum_{i=1}^{m} \sum_{j=1}^{m} b_{i j} x_{i} x_{j}+\cdots,
$$

где параметр $m$ определяет порядок модели.

Рассмотрим основные этапы МГУА. Главная идея метода заключается в разбиении экспериментальных данных на две части - обучающую и проверочную 
выборки, обозначаемые как $N_{A}$ и $N_{B}$ (количество наблюдений в каждой из частей обозначим как $n_{A}$ и $n_{B}$ соответственно).

На основании обучающей выборки $N_{A}$ строятся выражения по типу формулы (1), и затем при помощи метода наименьших квадратов определяются коэффициенты $b_{i}$. После чего для каждой модели рассчитывается один или несколько внешних критериев, смысл которых оценить её адекватность и работоспособность путем испытания на проверочной части экспериментальных данных $N_{B}$. В литературе, посвященной МГУА [14-17, 19-22], исследуется достаточно большое количество критериев, но наиболее распространёнными из них являются критерии регулярности $K_{r e g}$ и несмещённости $K_{u n b}$.

Критерий регулярности $K_{r e g}$ показывает, насколько построенная зависимость отклоняется от экспериментальных данных на проверочной части выборки, и описывается следующей формулой:

$$
K_{r e g}=\sum_{i=1}^{n_{B}}\left(Y_{i}\left(N_{B}\right)-Y_{i}^{*}\left(N_{B}\right)\right)^{2},
$$

где $Y_{i}\left(N_{B}\right)$ - экспериментальные значения зависимой переменой, попавшие в проверочную часть данных; $Y_{i}^{*}\left(N_{B}\right)$ - значения, полученные путем подстановки значений регрессоров из множества $N_{B}$ в построенную модель

Для расчёта критерия несмещённости $K_{\text {unb }}$ строиться вспомогательная модель того же порядка, что и проверяемая, но коэффициенты этой модели проверяются на основании выборки $N_{B}$. Таким образом, значение $K_{\text {unb }}$ определяется по следующей формуле:

$$
K_{u n b}=\sum_{i=1}^{n_{B}}\left(Y_{i}^{*}\left(N_{B}\right)-\widetilde{Y}_{l}\left(N_{B}\right)\right)^{2},
$$

где $\widetilde{Y}_{l}\left(N_{B}\right)$ - значения зависимой переменной, полученные из вспомогательной модели путем подстановки значений регрессоров из множества $N_{B}$.

На практике обычно используют следующий подход для выбора оптимальной модели из имеющихся: сперва производят расчет критерия регулярности для всех проверяемых моделей, после чего отбирают несколько имеющих наименьшее значение $K_{r e g}$, затем производят вычисление $K_{\text {unb }}$ для отобранных моделей; модель, имеющая минимальное значение $K_{u n b}$, определяется как оптимальная [14]. Одним из замечательных свойств МГУА является то, что значения критериев (2) и (3) достигают минимума при определенной сложности моделей и далее начинают возрастать [15], что позволяет определить оптимальный порядок модели на основании имеющихся данных.

Как показывает практика применения данного метода [15], в ряде случаев необходимо ввести дополнительные переменные $x_{m+1}, \ldots x_{m+k}$, которые являются какой-либо нелинейной комбинацией регрессоров, не учитываемой в модели (1). В нашем случае, исходя из предварительных исследований, введем переменные $x_{3}=\frac{x_{1}}{x_{2}}$ и $x_{4}=\sqrt{\frac{x_{1}}{x_{2}}}$.
Исходя из малого количества наблюдений, ограничимся рассмотрением моделей не выше второго порядка. Также при построении будем оперировать только тремя переменными, а именно использовать набор $\left(x_{1} ; x_{2} ; x_{3}\right)$, либо $\left(x_{1} ; x_{2} ; x_{4}\right)$. Таким образом, методика применения МГУА к задаче построения регрессионной модели в данной работе выглядит следующим образом:

1) выбор зависимой переменной $y$;

2) выбор набора регрессоров, на которых будет строиться модель;

3) разбиение табл. 1 на две части $N_{A}$ и $N_{B}$;

4) назначение порядка $m$, определяющего сложность модели (начиная от $m=0$ );

5) вычисление коэффициентов модели по методу наименьших квадратов на основании выборки $N_{A}$;

6) вычисление коэффициентов сопряженной модели по методу наименьших квадратов на основании выборки $N_{B}$;

7) расчёт критериев (2) и (3) для данной модели;

8) увеличение порядка модели на 1 и переход к п. 5;

9) после обработки всех исследуемых моделей сортировка полученных результатов по критерию $K_{r e g}$;

10) выбор среди пяти лучших моделей по критерию $K_{r e g}$ минимальной по критерию $K_{u n b}$.

Данная методика обработки результатов была реализована в среде Matlab с использованием встроенных инструментов данной среды.

На рис. 2 приведен фрагмент листинга кода разработанной программы.

Основным инструментом в данном случае является встроенная функция fittm() [20], которая осуществляет расчет коэффициентов искомой модели по методу наименьших квадратов. Отметим также, что в данном случае сложность модели определяется тремя переменными $i, j, k$, каждая из которых задает максимальную степень соответствующего регрессора, задействованного в модели. Например, значениям $i=1, j=1, k=2$ будет соответствовать модель $y=b_{0}+b_{1} x_{1}+b_{2} x_{2}+b_{3} x_{3}+b_{4} x_{3}^{2}+b_{5} x_{1} x_{3}+b_{6} x_{2} x_{3}$.

Представим результаты обработки в виде табл. 7 .

Таблица 7. Результаты обработки

\begin{tabular}{|c|c|c|}
\hline $\begin{array}{c}\text { Исследуемая } \\
\text { переменная } \\
\text { Study variable }\end{array}$ & $\begin{array}{c}\text { Набор } \\
\text { регрессоров } \\
\text { Set of } \\
\text { regressors }\end{array}$ & $\begin{array}{c}\text { Оптимальный поря- } \\
\text { док модели }(i, j, k) \\
\text { Optimal order of the } \\
\text { model }(i, j, k)\end{array}$ \\
\hline $\begin{array}{l}\text { Выход концентрата по Аu, } y_{1} \\
\text { Au concentrate output } y_{1}\end{array}$ & $\left(x_{1} ; x_{2} ; x_{3}\right)$ & $(1,0,2)$ \\
\hline $\begin{array}{l}\text { Выход хвостов по Au, } y_{2} \\
\text { Au tails output } y_{2}\end{array}$ & $\left(x_{1} ; x_{2} ; x_{3}\right)$ & $(1,0,0)$ \\
\hline $\begin{array}{l}\text { Выход концентрата по } \mathrm{Pb}, y_{3} \\
\mathrm{~Pb} \text { concentrate output, } y_{3}\end{array}$ & $\left(x_{1} ; x_{2} ; x_{4}\right)$ & $(1,1,1)$ \\
\hline $\begin{array}{l}\text { Выход хвостов по } \mathrm{Pb}, y_{4} \\
\mathrm{~Pb} \text { tails output, } y_{4}\end{array}$ & $\left(x_{1} ; x_{2} ; x_{4}\right)$ & $(2,0,0)$ \\
\hline
\end{tabular}

Table 7. Processing results

Таким образом, для каждой из переменных можно написать модель регрессии и определить коэффициенты по методу наименьших квадратов с помощью встроенной функции fitlm() языка MatLab. 


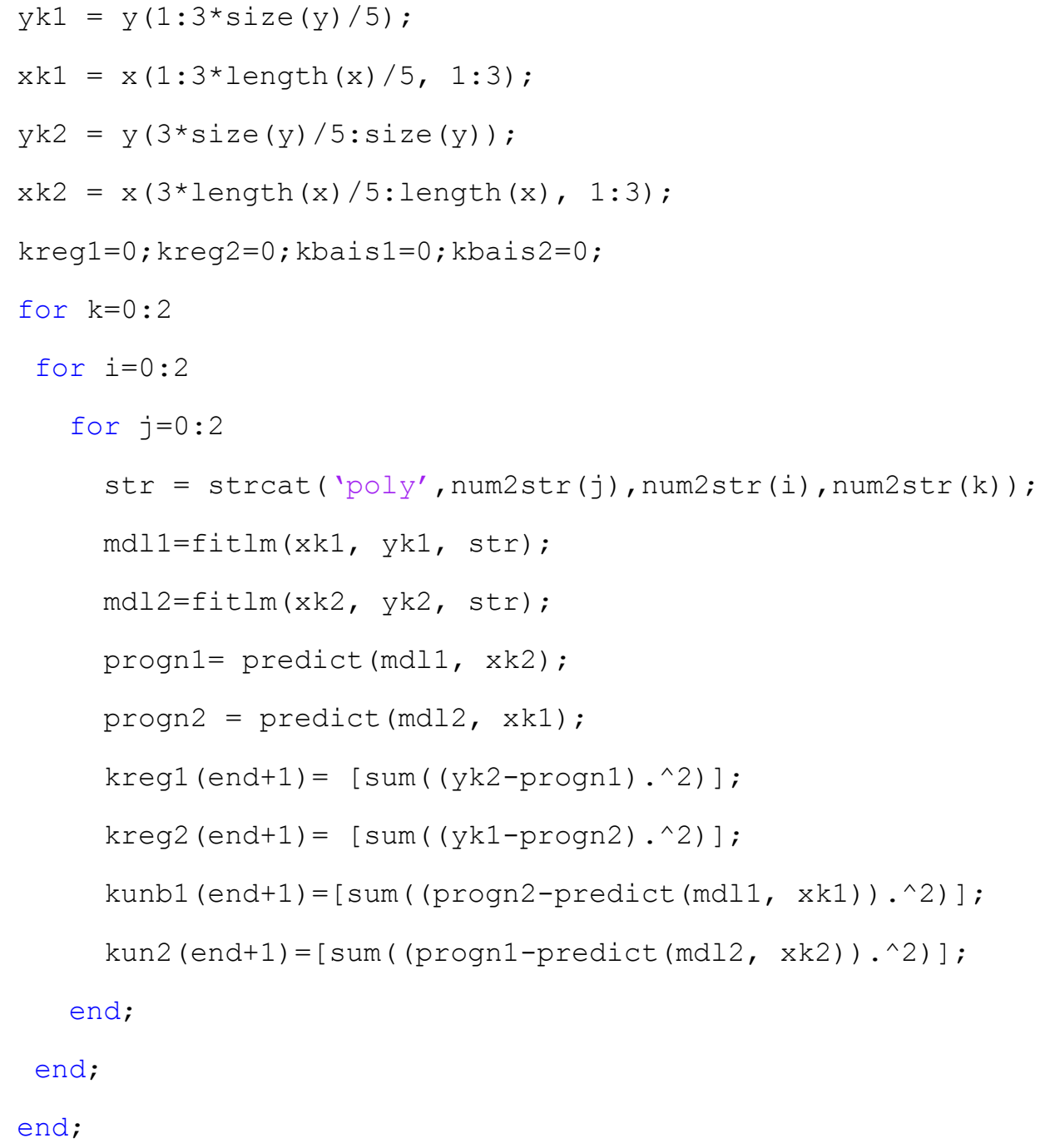

Puc. 6. Фрагмент кода в среде Matlab

Fig. 6. Code snippet in Matlab

Так, для содержания в концентрате по Аu имеем, что искомая зависимость имеет вид:

$$
\begin{aligned}
& y_{1}=b_{0}+b_{1} x_{1}+b_{2} x_{3}+b_{3} x_{3}^{2}+b_{4} x_{1} x_{3}= \\
& =b_{0}+b_{1} x_{1}+b_{2} \frac{x_{1}}{x_{2}}+b_{3}\left(\frac{x_{1}}{x_{2}}\right)^{2}++b_{4} \frac{x_{1}^{2}}{x_{2}} .
\end{aligned}
$$

Или, конкретизируя параметры $b_{i}$, получим:

$$
y_{1}=-48,85+1,6 x_{1}+21,9 \frac{x_{1}}{x_{2}}-0,65\left(\frac{x_{1}}{x_{2}}\right)^{2}-0,16 \frac{x_{1}^{2}}{x_{2}} \text {. }
$$

Из вышесказанного можно заключить, что содержание в концентрате по Аu на данной установке прямо пропорционально расходу и квадрату расхода флюидизационной воды и имеет обратную зависимость от времени открытия пережимных клапанов.

Для содержания в хвостах зависимость принимает вид:

$$
y_{2}=b_{0}+b_{1} x_{1}=1,31+0,004 x_{1} .
$$

Содержание в хвостах по $\mathrm{Au}$ слабо зависит от настраиваемых параметров, что позволяет сделать вывод об их постоянной величине для данной установки.

Содержание концентрата по $\mathrm{Pb}$ :

$$
\begin{aligned}
y_{3}= & b_{0}+b_{1} x_{1}+b_{2} x_{2}+b_{3} x_{4}= \\
= & b_{0}+b_{1} x_{1}+b_{2} x_{2}+b_{3} \sqrt{\frac{x_{1}}{x_{2}}} .
\end{aligned}
$$

После расчета параметров $b_{i}$ имеем:

$$
y_{3}=7,2+0,04 x_{1}-0,06 x_{2}-3,99 \sqrt{\frac{x_{1}}{x_{2}}} .
$$

Полученная регрессионная модель показывает, то содержание концентрата по свинцу слабо зависит от самих настраиваемых параметров $x_{1}$ и $x_{2}$, однако имеет чётко выраженную обратно пропорциональную зависимость от их комбинации.

Для выхода хвостов по свинцу имеем:

$$
y_{4}=b_{0}+b_{1} x_{1}+b_{2} x_{1}^{2}=6,26-0,34 x_{1}+0,005 x_{1}^{2} \text {. }
$$

Полученный результат для содержания в хвостах по $\mathrm{Pb}$ достаточно хорошо согласуется с аналогичным итогом для хвостов по Аи и также слабо зависит от настраиваемых параметров, что позволяет судить о постоянстве данной величины для данной установки. 


\section{Выводы}

На основании проведенных исследований установлена принципиальная возможность эффективного применения технологии CVD в качестве гравитационного обогащения. Результаты концентратора CVD значительно превосходят в эффективности отсадочные машины.

В результате работы сформирована простая и эффективная методика обработки результатов тестов обогатительного оборудования, базирующаяся на методе группового учета аргументов, реализованная в среде MatLAB.

На основании многочисленных вычислительных экспериментов построены статистически значимые модели, описывающие зависимость содержания $\mathrm{Au}$ и $\mathrm{Pb}$ в хвостах и концентрате от различных настраиваемых параметров оборудования, обоснована их эффективность, позволяющая применять данные модели в дальнейших исследованиях по применению CVD

\section{СПИСОК ЛИТЕРАТУРЫ}

1. Чмыхалова С.В. Влияние снижения качества и изменчивости руды на ресурсноэкологические показатели горного производства (на примере АО «Апатит») // Горный информационно-аналитический бюллетень (научно-технический журнал). 2018. - № 2. - С. 73-80.

2. Агабалян А.Ю. Технико-экономическое обоснование полноть извлечения металлов в концентрат // Вестник Национального политехнического университета Армении. Металлургия, материаловедение, недропользование. - 2016. - № 2. - С. 97-108

3. Шумилова Л.В., Костикова О.С. Анализ влияния вещественного состава руды серебро-полиметаллического месторождения «Гольцовое» на показатели обогащения // Вестник Забайкальского государственного университета. - 2015. - № 5 (120). C. $46-55$.

4. Gibson B., Wonyen D.G., Chehreh Chelgani S. A review of pretreatment of diasporic bauxite ores by flotation separation // Minerals Engineering. - 2017. - № 114. - P. 64-73

5. Chanturiya V.A. Innovation-based processes of integrated and high-level processing of natural and technogenic minerals in Russia // IMPC $2018-29^{\text {th }}$ International Mineral Processing Congress. - Moscow, Russia, 2019. - P. 2-12.

6. Мязин В.П., Литвинцева В.И. Изыскание новых селективных реагентов для повышения эффективности флотации свинцовоцинковых руд Ново-широкинского месторождения // Вестник Забайкальского государственного университета. - 2017. T. 23. - № 2. - C. 4-15.

7. Investigation of two-stage depressing by using hydrophilic polymer to improve the process of fluorite flotation / C. Zhang, W. Sun Y. Hu, Q. Guan, J. Gao // Journal of Cleaner Production. - 2018. V. 193. - P. 228-235.

8. The current state and future directions of percolation leaching in the Chinese mining industry: Challenges and opportunities / I.M.S.K. Ilankoon, Y. Tang, Y. Ghorbani, X. Deng, D. McBride // Minerals Engineering. - 2018. - V. 125. - P. 206-222.

9. Исследование обогатимости полиметаллической руды месторождения Забайкальского края / П.К. Федотов, А.Е. Сенченко, К.В. Федотов, А.Е. Бурдонов // Обогащение руд. - 2019. № 3. - C. 4-15.

10. IT enabled platforms for integrated design and optimization of mining operations and mineral processing plants / B.P. Pradip Gautham, S. Reddy, B. Rai, V. Runkana // IMPC $2018-29^{\text {th }}$ In- технологии. Установленная статистически значимая зависимость между содержанием в концентре и настраиваемыми параметрами позволяет проводить предварительные расчеты по эффективности внедрения CVD технологии на горно-обогатительных предприятиях

На основании разработанных моделей установлен факт отсутствия зависимости между содержанием в хвостах на обеих установках и временем открытия пережимных клапанов.

Анализ полученных данных показал, что наиболее эффективной схемой применения технологии Knelson CVD является установка данных концентраторов во вторичном цикле измельчения. В результате проведения тестов на сливе мельницы были получены более высокие показатели извлечения, коэффициента концентрации и содержания целевых металлов в концентрате, в сравнении с показателями, полученными на сливе гидроциклона.

ternational Mineral Processing Congress. - Moscow, Russia, 2019. - P. 3475-3488.

11. Montiel L., Dimitrakopoulos R. Simultaneous stochastic optimization of production scheduling at Twin Creeks Mining Complex, Nevada // Mining Engineering. - 2018. - V. 70 (12). - P. 48-56.

12. Optimization of gold ore Sumbawa separation using gravity method: Shaking table / A.D. Ferdana, H.T.B.M. Petrus, I.M. Bendiyasa, F. Hamada, T. Sachiko // AIP Conference Proceedings. 2018. - 020070 .

13. Применение Knelson CVD-технологии для обогащения золото-свинцовой руды / В.В. Пелих, В.М. Салов, А.Е. Бурдонов, Н.Д. Лукьянов // Обогащение руд. - 2019. - № 1. -С. 3-11.

14. Ивахненко А.Г. Индуктивный метод самоорганизации сложных систем. - Киев: Наукова думка, 1982. - 296 с.

15. Ивахненко А.Г., Мюллер Й.А. Самоорганизация прогнозирующих моделей. - Киев: Техніка, 1985. - 223 с.

16. Баласанян С.Ш., Геворгян Э.М. Сравнительный анализ методов регрессии и метода группового учета аргументов при моделировании процессов переработки полезных ископаемых // Известия Томского политехнического университета. - 2016. Т. 327 - № 4. - C. 23-34.

17. Баласанян С.Ш. Метод стратифицированной формализации сложных технологических систем со многими состояниями // Известия Томского политехнического университета. Инжиниринг георесурсов. - 2016. - Т. 327. - № 1. - С. 6-18.

18. Стрижов В.В., Крымова Е.А. Методы выбора регрессионных моделей. - М.: ВЦ РАН, 2010. - 60 с

19. Application of GMDH and variable co-integration theory in powerload forecasting / J. Gu, L.L. Chu, Y.J. Zhang, W.G. Shi // Power Syst. Prot. Control. - 2010. - № 38. - P. 80-85.

20. Onwubolu G. GMDH-methodology and implementation in MATLAB. $1^{\text {st }}$ ed. - London, UK: Imperial College Press, 2016. $74 \mathrm{p}$.

21. Najafzadeh M., Saberi-Movhed F., Sarkamaryan S. NF-GMDHbased self-organized systems to predict bridge pier scour depth under debris flow effects // Mar. Georesour. Geotechnol. - 2017. № 36. - P. 589-602.

22. Сергеев В.Л., Нгуен Т.Х.Ф. Модели и алгоритмы адаптивной интерпретации результатов комбинированных газогидродинамических исследований интеллектуальных скважин // Известия Томского политехнического университета. Инжиниринг георесурсов. - 2018. - Т. 329. - № 10. - С. 67-75. 
Информация об авторах

Пелих В.B., инженер по наладке систем управления и монтажу технологического оборудования ООО «ФЛСмидт Рус» в г. Иркутске.

Салов В.М., кандидат технических наук, профессор, профессор кафедры автоматизации технологических процессов, Иркутский национальный исследовательский технический университет.

Бурдонов $\boldsymbol{A . E}$., кандидат технических наук, доцент кафедры обогащения полезных ископаемых и охраны окружающей среды, Иркутский национальный исследовательский технический университет.

Лукьянов Н.Д., кандидат технических наук, доцент кафедры автоматизированных систем, Иркутский национальный исследовательский технический университет. 
UDC $622.7+541.183+66-5$

\title{
ESTABLISHMENT OF TECHNOLOGICAL DEPENDENCE OF KC-CVD6 CONCENTRATOR OPERATION BY MEANS OF THE ARGUMENT GROUP ACCOUNTING METHOD
}

\author{
Vladislav V. Pelikh ${ }^{1}$ \\ salov@istu.edu
}

\author{
Valery M. Salov², \\ salov@istu.edu
}

\author{
Alexander E. Burdonov², \\ slimbul@inbox.ru \\ Nikita D. Lukyanov², \\ Lukyanov.n@gmail.com \\ 1 FLSmidt Rus in Irkutsk, \\ 1/4, Oktyabrskoy revolyutsii street, Irkutsk, 664007, Russia. \\ 2 Irkutsk National Research Technical University, \\ 83, Lermontov street, Irkutsk, 664074, Russia.
}

The relevance of the research is caused by the acquisition of new knowledge on optimization of the technological parameters of centrifugal concentrator operation using the Knelson CVD technology, namely: setting the frequency of opening the valves and the time during which the valves remain open.

The aim of the research is a statistical analysis of the results of semi-industrial studies of the Knelson CVD technology applicability on various products of the factory, using the method of group accounting of arguments, to derive dependencies between the concentrate yield and tunable parameters, which allow preliminary calculations of the effectiveness of implementation of this technology at the processing enterprises.

Objects: products obtained as a result of hydrocyclone operation, as well as the technological parameters of the centrifugal concentrators. Methods: general methods of mathematical statistics, in particular, regression analysis methods aimed at constructing statistically significant models describing the dependence of a variable on the set of regressors. Also, along with the classical apparatus of regression analysis, the method of group accounting of arguments was used. The main idea of the method is to build a set of models of a given class, and to select optimal one among them.

Results. The authors have developed the effective method for processing the results of tests carried out on the processing equipment, based on the method of group accounting of arguments. Based on the obtained data, statistically significant models were constructed, describing the dependence of the content in tails and the concentrate on valuable components on various adjustable equipment parameters, and their effectiveness was justified, which makes it possible to apply these models in further studies on the application of CVD technology. The paper demonstrates the application of the method of group accounting of arguments using the example of constructing polynomial regression models containing non-linear combinations of regressors.

\section{Key words:}

Knelson CVD, gravity concentration, gold, lead, extraction, tails, technology.

\section{REFERENCES}

1. Chmykhalova S.V. The impact of reducing the quality and variability of ore on resource-environmental indicators of mining production (for example, JSC «Apatite»). Mining informational and analytical bulletin, 2018, no. 2, pp. 73-80. In Rus.

2. Agabalyan A.Yu. Tekhniko-ekonomicheskoe obosnovanie polnoty izvlecheniya metallov $\mathrm{v}$ kontsentrat [A feasibility study on the complete extraction of metals into concentrate]. Herald of the $\mathrm{Na}$ tional Polytechnic University of Armenia. Metallurgy, materials science, subsoil use, 2016, no. 2, pp. 97-108.

3. Shumilova L.V., Kostikova O.S. Analysis of the influence of the material composition of the ore of the silver-polymetallic deposit «Goltsovoe» on the enrichment indicators. Transbaikal State University Journal, 2015, no. 5 (120), pp. 46-55. In Rus.

4. Gibson B., Wonyen D.G., Chehreh Chelgani S. A review of pretreatment of diasporic bauxite ores by flotation separation. Minerals Engineering, 2017, no. 114, pp. 64-73.

5. Chanturiya V.A. Innovation-based processes of integrated and high-level processing of natural and technogenic minerals in Russia. IMPC $2018-29^{\text {th }}$ International Mineral Processing Congress. Moscow, Russia, 2019. pp. 3-12.

6. Myazin V.P., Litvintseva V.I. Search for new selective reagents to increase the efficiency of flotation of lead-zinc ores of the Novo-
Shirokinsky deposit. Transbaikal State University Journal, 2017, vol. 23, no. 2, pp. 4-15. In Rus.

7. Zhang C., Sun W., Hu Y., Guan Q., Gao J. Investigation of twostage depressing by using hydrophilic polymer to improve the process of fluorite flotation. Journal of Cleaner Production, 2018, vol. 193 , pp. 228-235.

8. Ilankoon I.M.S.K., Tang Y., Ghorbani Y., Deng X., McBride D. The current state and future directions of percolation leaching in the Chinese mining industry: Challenges and opportunities. Minerals Engineering, 2018, vol. 125, pp. 206-222.

9. Fedotov P.K., Senchenko A.E., Fedotov K.V., Burdonov A.E. Study of the enrichment of polymetallic ore deposits of the TransBaikal Territory. Obogashchenie Rud, 2019, no. 3, pp. 4-15. In Rus.

10. Pradip, Gautham, B.P., Reddy S., Rai B., Runkana V. IT enabled platforms for integrated design and optimization of mining operations and mineral processing plants. IMPC $2018-29^{\text {th }}$ International Mineral Processing Congress. Moscow, Russia, 2019. pp. $3475-3488$.

11. Montiel L., Dimitrakopoulos R. Simultaneous stochastic optimization of production scheduling at Twin Creeks Mining Complex, Nevada. Mining Engineering, 2018, vol. 70 (12), pp. 48-56.

12. Ferdana A.D., Petrus H.T.B.M., Bendiyasa I.M., Hamada F., Sachiko T. Optimization of gold ore Sumbawa separation using 
gravity method: Shaking table. AIP Conference Proceedings, 2018, 020070.

13. Pelikh V.V., Salov V.M., Burdonov A.E., Lukyanov N.D. Application of Knelson CVD technology for beneficiation of gold-lead ore. Obogashchenie Rud, 2019, no. 1, pp. 3-11. In Rus.

14. Ivakhnenko A.G. Induktivny metod samoorganizatsii slozhnykh sistem [Inductive method of self-organization of complex systems] Kiyev, Naukova dumka Publ., 1982. 296 p.

15. Ivakhnenko A.G., Muller Y.A. Samoorganizatsyya prognoziruyushchikh modeley [Self-organization of forecasting models]. Kyev, Tekhnika Publ., 1985. 233 p.

16. Balasanyan S.Sh., Gevorgyan E.M. Comparative analysis of the regression methods and the method of group accounting of arguments in the modeling of mineral processing processes. Bulletin of the Tomsk Polytechnic University, 2016, vol. 327, no. 4, pp. 23-34 In Rus.

17. Balasanyan S.Sh. The method of stratified formalization of complex technological systems with many states. Bulletin of the Tomsk Polytechnic University. Geo Assets Engineering, 2016, vol. 327, no. 1 , pp. 6-18.
18. Strizhov V.V., Krymova E.A. Metody vybora regressionnykh modeley [Methods for choosing regression models]. Moscow, VTS RAN Publ., 2010. $60 \mathrm{p}$.

19. Gu J., Chu L.L., Zhang Y.J., Shi W.G. Application of GMDH and variable co-integration theory in power load forecasting. Power Syst. Prot. Control, 2010, vol. 38, pp. 80-85.

20. Onwubolu G. GMDH-methodology and implementation in MATLAB. ${ }^{\text {st }}$ ed. London, UK, Imperial College Press, 2016. 74 p.

21. Najafzadeh M., Saberi-Movhed F., Sarkamaryan S. NF-GMDHbased self-organized systems to predict bridge pier scour depth under debris flow effects. Mar. Georesour. Geotechnol., 2017, vol. 36, pp. 589-602.

22. Sergeev V.L., Nguyen T.H.F. Models and algorithms for adaptive interpretation of the results of combined gas-hydrodynamic studies of intelligent wells. Bulletin of the Tomsk Polytechnic University. Geo Assets Engineering, 2018, vol. 329, no. 10, pp. 67-75.

Received: 24 January 2020.

\section{Informaton about the authors}

Vladislav V. Pelikh, engineer for control system adjustment and technological equipment installation, FLSmidt Rus in Irkutsk.

Valery M. Salov, Cand. Sc., professor, Irkutsk National Research Technical University.

Alexander E. Burdonov, Cand. Sc., associate professor, Irkutsk National Research Technical University.

Nikita D. Lukyanov, Cand. Sc., associate professor, Irkutsk National Research Technical University. 